\title{
EDITORIAL
}

\section{Communities at the centre}

\author{
Nature Reviews Microbiology celebrates its 15th anniversary and with it the microbiology \\ community, many of whom have contributed directly and indirectly to the journal.
}

15 years of Nature Reviews Microbiology! And what an eventful 15 years it has been. Microbiology is flourishing since 2003, the number of microbiology articles published each year has almost doubled, at least if you ask Web of Science for the number of articles that fall in their microbiology category.

One could lament the publish-or-perish climate or the publishing landscape, but much of this increase is due to new discoveries and genuine growth of knowledge in the field. Open a general science journal and you will certainly find microbiology articles - topics, such as epidemics and pandemics, emerging infectious diseases, the antimicrobial resistance crisis, CRISPR-Cas and last but not least the microbiome, are everywhere. Mind you, even in newspapers these topics often crop up. Although, this editor still did a double take when her non-scientist, 75-year old aunt enquired about phage therapy and antibiotic resistance. Similarly, Nancy Keller, who has written a Review about fungal secondary metabolites for the journal in the past, recounted that she frequently gets asked about the role of the microbiome and its health benefits by non-scientist friends and relatives (see an interview with her on our community site).

To celebrate our anniversary and to give our readers a glimpse behind the curtains, we asked a few of our past authors and editors about their views on microbiology, the journal and their experiences of working with us. The importance of the microbiome came up time and again. The omnipresence, diversity and versatility of microbial communities are staggering, and there is still much to be learned. It has become clear that microorganisms can rarely be viewed in isolation and that understanding their interactions and surroundings is key. Similarly, today's microbiologists can rarely work in isolation - research often is interdisciplinary and requires the input and collaboration of specialists with different skills and backgrounds.

As a Reviews journal, we hope to foster connections between different research communities and to help establish a common language that is accessible for readers at all levels of expertise and with varied backgrounds. The authors who we have interviewed have been exceedingly courteous about our editorial input, but we are well aware that receiving an article after it has been edited by us might come as shock. Our authors are experts and many are accomplished writers, but there is always some jargon to scrub, some waffle to tighten or some gaps to fill. Also, as our authors are experts, it sometimes can be hard to write for a general microbiology audience. A side note - if you ever write for us and find the comment "please explain for an unfamiliar reader or a non-specialist" in the edited manuscript, this can be translated to "please explain for this editor, who has looked it up on Wikipedia but still has trouble understanding". Digression over, if you are interested in what our authors, past editors and, of special note, our art editor have to say about microbiology and the journal, have a look at the interviews on our community site. Although be warned, we have asked them for their favourite articles in the journal and there is a high risk of spending an inordinate amount of time in our archives rediscovering some old, new and hidden gems, as we did when we went through their answers.

Nature Reviews Microbiology certainly is a community effort, and we do not want to miss the opportunity to thank our authors, peer reviewers, readers, the wider staff who support us, conference organizers, inspiring presenters, skilled seminar speakers, scientists who welcome us to their labs for visits and all the other people who make this journal what it is. We are very grateful for all your support, effort and acceptance! Speaking in microbiome metaphors, not all relationships are commensal or symbiotic and there certainly is competition, maybe even cheating. As every other organism, we adapt and evolve and we look forward to what the next 15 years will bring for the journal and the field of microbiology.

Last but not least, we also would like to use this opportunity to reflect on the roots of microbiology, when van Leeuwenhoek observed 'microscopic organisms' for the first time. To acknowledge how far microbial imaging has come and how sophisticated novel technologies are enabling the visualization of microbial structures and processes, we are launching a new series of short commentaries that discuss outstanding recent primary papers. Our anniversary issue contains the first of these 'Under the Lens' articles (see page 584), which are penned by microbial imaging specialists at the University of Oxford, UK.

Happy 15th, we are filled with gratitude and excitement to be part of the microbiology community! 